hep-th/0412066

IPPP $/ 04 / 82$

$\mathrm{DCPT} / 04 / 164$

\title{
Bottom Up Meets Heterotic Strings ${ }^{1}$
}

\author{
Stefan Förste ${ }^{a}$ and Akın Wingerter ${ }^{b}$ \\ ${ }^{a}$ Institute for Particle Physics Phenomenology (IPPP) \\ South Road, Durham DH1 3LE, United Kingdom \\ ${ }^{b}$ Physikalisches Institut der Universität Bonn \\ Nussallee 12, 53115 Bonn, Germany
}

\begin{abstract}
In this talk we argue that a certain class of heterotic orbifolds can be the underlying fundamental theory for recently studied field theory GUTs. In addition we demonstrate that symmetric heterotic $\mathbb{Z}_{2} \times \mathbb{Z}_{2}$ orbifolds can give rise to three generation models if quantised Wilson lines are switched on.
\end{abstract}

\footnotetext{
${ }^{1}$ Based on talks given by A. Wingerter at String Phenomenology 2004, Ann Arbor, 1-6 August 2004, and by S. Förste at the 37th International Symposium Ahrenshoop on the Theory of Elementary Particles, Berlin, 23-27 August 2004.
} 


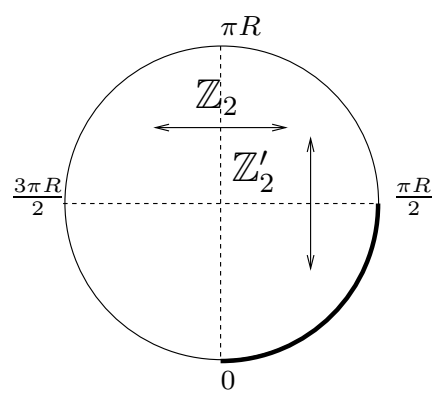

Figure 1: The double arrows indicate the action of $\mathbb{Z}_{2} \times \mathbb{Z}_{2}^{\prime}$ on the circle. Any point on the circle can be mapped into the right lower quarter segment - the fundamental domain is an interval of length $\pi R / 2$.

\section{Introduction}

Recently it was realised by model builders that the concept of extra dimensions leads to phenomenologically interesting models where the standard model gauge group merges into a grand unified group only after an extra dimension opens up. This class of models we view as bottom up theories. We will briefly summarise the concept in section one.

In the remaining and main part of the talk we focus on compactifications of heterotic $\mathrm{E}_{8} \times \mathrm{E}_{8}$ strings on orbifolds and argue that $\mathbb{Z}_{N} \times \mathbb{Z}_{M}$ orbifolds naturally provide the picture obtained in the bottom up approach. For simplicity we restrict our attention to $\mathbb{Z}_{2} \times \mathbb{Z}_{2}$ orbifolds. In order to reduce the number of generations to three, Wilson lines are required. We demonstrate at an example that in this way one can obtain three generation models.

We should emphasise that the historical order has been reversed in this talk. Orbifolds have been studied first (and long time ago) in the context of string theory and only recently were 'rediscovered' by model builders.

This talk is based on results reported in [1].

\section{Bottom Up: Orbifold GUTs}

The concept of extra dimensions allows for the possibility that a grand unified gauge group is realised only in a higher dimensional space. Let us briefly illustrate this at a simple example [2]. We consider the case of one extra dimension which is compactified on an orbicircle $S^{1} / \mathbb{Z}_{2} \times \mathbb{Z}_{2}^{\prime}$, see figure 1] The orbicircle is equivalent to an interval of length $\pi R / 2$. As our five dimensional theory we take the minimal supersymmetric extension of $\mathrm{SU}(5)$ gauge theory. If the extra dimension were compactified on a circle we would get $\mathcal{N}=2$ supersymmetry in four dimensions. For the interval the situation is different. For a point in the bulk of the interval the $\mathbb{Z}_{2} \times \mathbb{Z}_{2}^{\prime}$ relates the value of a field at that point to the value at the image points. The ends of the interval are however invariant under elements of the orbifold action and hence we obtain a projection there. In particular one can embed the orbifold action into the field space such that $\mathbb{Z}_{2}$ projects the $\mathcal{N}=2$ gauge multiplet 
to an $\mathcal{N}=1$ multiplet and $\mathbb{Z}_{2}^{\prime}$ projects the $\mathrm{SU}(5)$ multiplet to an $\mathrm{SU}(3) \times \mathrm{SU}(2) \times \mathrm{U}(1)$ multiplet. Massless fields in four dimensions are obtained from modes which do not depend on the extra dimension and hence we obtain $\mathcal{N}=1 \mathrm{SU}(3) \times \mathrm{SU}(2) \times \mathrm{U}(1)$ gauge theory in four dimensions. In addition there are massive states whose mass is an integer times the compactification scale. The standard model matter is put into the five dimensional model either at the fixed points or into the bulk (for more details see [2]).

Orbifolds with one or two extra dimensions have received a lot of attention in the recent past, e.g. [2-7]. (For a review and more references see [8].) The reason is that these constructions allow to keep attractive features of conventional GUTs (e.g. prediction of hypercharge) while avoiding typical problems (e.g. doublet-triplet splitting, proton decay, unrealistic fermion mass matrices). However, one problem of the orbifold GUTs is that they allow for lots of freedom and one can construct many models. The number of extra dimensions is not fixed. The grand unified group is a matter of choice. The localisation of charged matter is not predicted but put in by hand. That leads to the desire to construct these phenomenologically attractive class of models from a more fundamental theory. In the following we will demonstrate that $\mathbb{Z}_{N} \times \mathbb{Z}_{M}$ heterotic $\mathrm{E}_{8} \times \mathrm{E}_{8}$ models naturally contain an intermediate orbifold GUT picture. (The same is true for $\mathbb{Z}_{N}$ orbifolds if $N$ is not a prime number whereas for prime numbers one always has an $\mathrm{E}_{8} \times \mathrm{E}_{8}$ symmetry in the bulk.)

\section{$3 \quad$ Heterotic Orbifolds}

The heterotic $\mathrm{E}_{8} \times \mathrm{E}_{8}$ string theory is a consistent string theory in $9+1$ dimensions [9]. The massless spectrum consists of gravity, $\mathrm{E}_{8} \times \mathrm{E}_{8}$ gauge fields ${ }^{1}$ and the susy partners of minimal supersymmetry in ten dimensions. $\mathrm{E}_{8}$ is an exceptional Lie algebra with eight Cartan generators $H_{I}, I=1, \ldots, 8$ and 240 root generators $E_{\alpha}$. Since we do not live in $9+1$ but in $3+1$ dimensions we have to compactify six dimensions. The simplest choice would be a six dimensional torus $T^{6}$. In the following we will describe $T^{6}$ as the direct product of three two dimensional tori $T^{2}$. Each $T^{2}$ can be identified with a lattice in a complex plane where opposite edges of a cell within the lattice are identified. If we just compactify on $T^{6}$ we obtain an $\mathcal{N}=4$ supersymmetric field theory in four dimensions. This is not a realistic model since e.g. it predicts gauge couplings which are independent of the energy scale. It would be more realistic to have $\mathcal{N}=1$ supersymmetry. (No supersymmetry agrees of course also with experimental data, but we focus on $\mathcal{N}=1$ in this talk.) In order to reduce the number of supersymmetries one replaces $T^{6}$ by an orbifold of $T^{6}$. This idea is known for a rather long time, in the original papers $[11,12]$ special attention has been given to the $T^{6} / \mathbb{Z}_{3}$ orbifold. Here, we will focus instead on $T^{6} / \mathbb{Z}_{2} \times \mathbb{Z}_{2}$ which should be viewed as a representative of the class $T^{6} / \mathbb{Z}_{N} \times \mathbb{Z}_{M}$ or $T^{6} / \mathbb{Z}_{N}$ with $N$ not prime ${ }^{2} . \mathbb{Z}_{2} \times \mathbb{Z}_{2}$ is the orbifold group acting on $T^{6}$. Points which are mapped onto each other by the orbifold group are identified. Our $\mathbb{Z}_{2} \times \mathbb{Z}_{2}$ orbifold group is generated by two elements. The first element acts

\footnotetext{
${ }^{1}$ For recent discussions of the $\mathrm{SO}(32)$ heterotic string see [10].

${ }^{2}$ In the free fermionic formulation, $\mathbb{Z}_{2} \times \mathbb{Z}_{2}$ orbifolds have been studied extensively by Faraggi and various collaborators, see e.g. [13].
} 


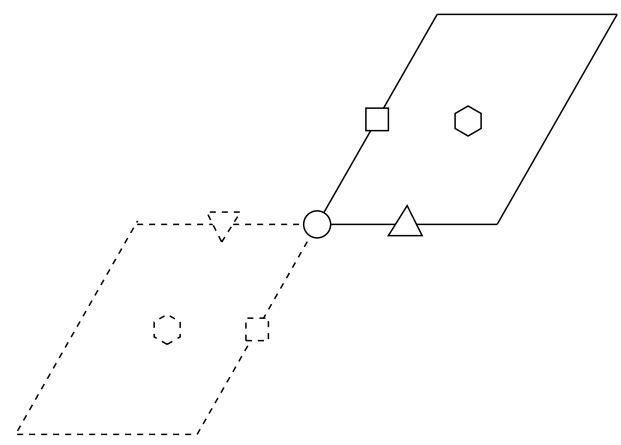

Figure 2: Fixed points on one $T^{2} / \mathbb{Z}_{2}$. The $T^{2}$ is obtained by identifying opposite solid lines. Dashed lines are $\mathbb{Z}_{2}$ images of solid lines.

as a simultaneous $180^{\circ}$ rotation on the first $\left(x^{4}+i x^{5}\right)$ and the second $\left(x^{6}+i x^{7}\right)$ complex plane and leaves the third plane $\left(x^{8}+i x^{9}\right)$ invariant. The second generator leaves the first plane invariant and acts as a $180^{\circ}$ rotation on the second and third plane. There is one more non trivial element in $\mathbb{Z}_{2} \times \mathbb{Z}_{2}$ which acts as a $180^{\circ}$ rotation on the first and third plane and leaves the second one invariant. This element is the product of the two generators. It is useful to associate the elements of the orbifold group with three dimensional vectors. The two generators correspond to

$$
v_{1}=\left(\frac{1}{2},-\frac{1}{2}, 0\right), v_{2}=\left(0, \frac{1}{2},-\frac{1}{2}\right) .
$$

This means for example that the first generator consists of a rotation by $\frac{1}{2} \cdot 2 \pi$ in the first plane, $-\frac{1}{2} \cdot 2 \pi$ in the second plane and $0 \cdot 2 \pi$ in the third plane.

For reasons to become clear in a moment the notion of a fixed point is a very important concept. Let us first look at only one $T^{2}$ in figure 2. The point at the origin (depicted as a circle) is invariant under the rotation and trivially fixed. All other fixed points (depicted as triangle, square and hexagon) differ from their images by a lattice shift and thus are identical on $T^{2}$. The qualitative difference between the origin and the other fixed points will be important later. There are twisted strings which are closed only when the orbifold identification is taken into account. The center of mass position of these strings is constrained to the location of a fixed point and its excitations corresponds to matter located at the fixed point. In the $\mathbb{Z}_{2} \times \mathbb{Z}_{2}$ orbifold each non trivial element leaves 16 tori fixed. (Recall that on one of the three tori the action is always trivial, the 16 counts the possible combinations of the two sets of four fixed points in the other two tori.) Since we have three non-trivial elements in the orbifold group this yields 48 fixed tori altogether.

The orbifold group acts not only on $T^{6}$ but will be also embedded into the gauge group. We use the so called shift embedding

$$
H_{I} \rightarrow H_{I}, \quad E_{\vec{\alpha}} \rightarrow e^{2 \pi i \vec{V}_{i} \cdot \vec{\alpha}} E_{\vec{\alpha}} .
$$


The 16 Cartan generators $H_{I}$ of $\mathrm{E}_{8} \times \mathrm{E}_{8}$ are left invariant. A 16 dimensional root vector of $\mathrm{E}_{8} \times \mathrm{E}_{8}$ is denoted by $\vec{\alpha}$ and $\vec{V}_{i}$ is a 16 component vector characterising how the $i$ th element of the orbifold group is embedded into the gauge group. String theory gives very restrictive consistency conditions on how the orbifold group can be embedded into the gauge group and it turns out that there are only five consistent choices for the $\mathbb{Z}_{2} \times \mathbb{Z}_{2}$ case [1].

In the rest of the talk we will restrict ourselves to the standard embedding which means that we copy $v_{i}$ into the first three components of $\vec{V}_{i}$ and set the remaining components to zero

$$
\vec{V}_{i}=(v_{i}, \underbrace{0, \ldots, 0}_{13}) .
$$

When constructing the invariant spectrum one finds at the massless level the gravity multiplet, the gauge multiplet and chiral multiplets in the untwisted sector, and more chiral multiplets in the twisted sector. In our example one finds among others (for details see $[1,14])$ an $\mathrm{E}_{6} \times \mathrm{U}(1)^{2} \times \mathrm{E}_{8}$ gauge multiplet and one 27 dimensional representation of $\mathrm{E}_{6}$ per fixed torus. This would lead to a model with 48 generations which is to much. In order to reduce the number of generations we need to lift the fixed point degeneracy.

The fixed point degeneracy is lifted when non trivial Wilson lines are present [15]. A Wilson line is a non zero vacuum expectation value for an internal gauge field component $A_{k}, k=4, \ldots, 9$. We choose the expectation value to lie in the Cartan subalgebra of $\mathrm{E}_{8} \times \mathrm{E}_{8}$. This is in general not invariant under the orbifold group (the embedding into the Cartan subalgebra is trivial but the action on the internal dimensions is non trivial). Therefore, the vacuum expectation value can only take discrete values such that the orbifold image and the vev differ by a (periodic) gauge transformation. The Wilson line is quantised. Let us look for example at ( $y$ is the fifth coordinate and $R_{5}$ the radius of the circle on which it is compactified)

$$
A_{5}=\frac{i}{R_{5}} \vec{a}_{5} \cdot \vec{H}=e^{-i \vec{a}_{5} \cdot \vec{H} y / R_{5}} \partial_{y} e^{i \vec{a}_{5} \cdot \vec{H} y / R_{5}},
$$

which is of the form $g^{-1} \partial_{y} g$ and looks like a pure gauge. This gauge is however not single valued under $y \rightarrow y+2 \pi R_{5}$. We translate the vacuum expectation value for $A_{5}$ into the rule that going once around the fifth direction induces the gauge transformation

$$
E_{\alpha} \rightarrow e^{2 \pi i \vec{a}_{5} \cdot \vec{H}} E_{\alpha} e^{-2 \pi i \vec{a}_{5} \cdot \vec{H}}=e^{2 \pi i \vec{a}_{5} \cdot \vec{\alpha}} E_{\alpha} .
$$

Note that this looks like the shift embedding (2). In our case, the quantisation condition on the Wilson line is that twice $a_{5}$ should lie in the $\mathrm{E}_{8} \times \mathrm{E}_{8}$ root lattice. Now the fixed point degeneracy is lifted since the fixed points are fixed under the combined action of an orbifold group element and a fixed point dependent $T^{6}$ lattice shift. We should also emphasise that string theory puts a set of consistency conditions on the Wilson lines [16].

This way we can easily find a toy model with three generations [1]. We use standard embedding and six non zero Wilson lines, e.g.

$$
a_{4}=(\underbrace{0, \ldots, 0}_{7}, 1,1, \underbrace{0, \ldots, 0}_{7}) \text {. }
$$




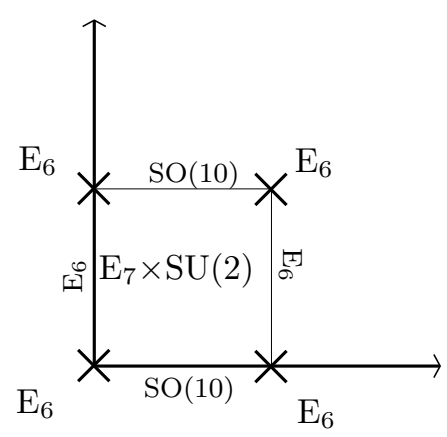

Figure 3: Orbifold GUT with underlying heterotic orbifold.

This Wilson line breaks the $\mathrm{E}_{6}$ to $\mathrm{SO}(10) \times \mathrm{U}(1)$ (from now on we do not discuss the second (hidden sector) $\mathrm{E}_{8}$ ). The other five Wilson lines have non zero entries only in the hidden sector $\mathrm{E}_{8}$ [1]. So, in the observable sector the gauge group is $\mathrm{SO}(10) \times \mathrm{U}(1)^{3}$. Because we have lifted the degeneracy of the fixed points with the help of the Wilson lines we now find three generations, i.e. three sixteen dimensional representations of $\mathrm{SO}(10)$ from the twisted sectors.

Now we show that our heterotic orbifold gives rise to the picture of a field theory orbifold given in the beginning of this talk. First we compactify the heterotic string on a $T^{4} / \mathbb{Z}_{2}$ where the $\mathbb{Z}_{2}$ is generated by one of the non trivial elements of $\mathbb{Z}_{2} \times \mathbb{Z}_{2}$ which we choose to be the second generator. This gives rise to a six dimensional model: four dimensional Minkowski space times $T^{2}$. The field theory orbifold is now obtained by replacing $T^{2}$ with $T^{2} / \mathbb{Z}_{2}$, where the $\mathbb{Z}_{2}$ is generated by the first generator of our $\mathbb{Z}_{2} \times \mathbb{Z}_{2}$. Now the gauge group in the bulk and the embedding of the orbifold group into the field space is not a matter of choice but given by the string theory construction. In the bulk there is an $\mathrm{E}_{7} \times \mathrm{SU}(2)$ ( $\times$ hidden sector) gauge group. At each fixed point of $T^{2} / \mathbb{Z}_{2}$ the orbifold group imposes projection conditions which break it to $\mathrm{E}_{6} \times \mathrm{U}(1)^{2}$. This looks as if the fixed points were degenerate. But for fixed points separated in the fourth direction the $\mathrm{E}_{6}$ is embedded differently into the bulk gauge group such that the overlap is $\mathrm{SO}(10)$ which is the gauge group in four dimensions, see figure 3 .

\section{Conclusions}

In this talk we have demonstrated that a certain class of heterotic string orbifolds naturally incorporate lower dimensional orbifold GUT models. In fact, one does not need to take the detour via orbifold GUTs but can formulate rules for string phenomenology straightaway [17]. It looks promising to find phenomenologically interesting models by investigating the orbifold groups $\mathbb{Z}_{N} \times \mathbb{Z}_{M}$, or $\mathbb{Z}_{K}$ with $K$ non prime. Wilson lines are needed in order to obtain a realistic number of generations. Even though heterotic orbifold constructions have been known for quite some time the above class has found attention only recently [18], [1], [19]. 


\section{Acknowledgments}

We would like to thank Hans Peter Nilles and Patrick Vaudrevange for a pleasant collaboration leading to the results presented in this talk. We would like to thank the organisers of String Phenomenology 2004 and the organisers of the 37th International Symposium Ahrenshoop on the Theory of Elementary Particles for creating a pleasant and stimulating atmosphere. This work was partially supported by the European community's Marie Curie programs MRTN-CT-2004-503369 "Quest for Unification" and MRTN-CT2004-005104 "Forces Universe".

\section{References}

[1] S. Förste, H. P. Nilles, P. K. S. Vaudrevange and A. Wingerter, Phys. Rev. D 70 (2004) 106008 arXiv:hep-th/0406208.

[2] Y. Kawamura, Prog. Theor. Phys. 105 (2001) 999 arXiv:hep-ph/0012125; Y. Kawamura, Prog. Theor. Phys. 105 (2001) 691 arXiv:hep-ph/0012352.

[3] G. Altarelli and F. Feruglio, Phys. Lett. B 511 (2001) 257 arXiv:hep-ph/0102301.

[4] L. J. Hall and Y. Nomura, Phys. Rev. D 64 (2001) 055003 arXiv:hep-ph/0103125.

[5] T. Kawamoto and Y. Kawamura, arXiv:hep-ph/0106163.

[6] A. Hebecker and J. March-Russell, Nucl. Phys. B 613 (2001) 3 arXiv:hep-ph/0106166.

[7] T. Asaka, W. Buchmüller and L. Covi, Phys. Lett. B 523 (2001) 199 arXiv:hep-ph/0108021.

[8] L. J. Hall and Y. Nomura, Annals Phys. 306 (2003) 132 arXiv:hep-ph/0212134.

[9] D. J. Gross, J. A. Harvey, E. J. Martinec and R. Rohm, Nucl. Phys. B 256 (1985) 253.

[10] J. Giedt, Nucl. Phys. B 671 (2003) 133 arXiv:hep-th/0301232; J. Giedt, arXiv:hep-ph/0402201; K. S. Choi, S. G. Nibbelink and M. Trapletti, arXiv:hep-th/0410232,

[11] L. J. Dixon, J. A. Harvey, C. Vafa and E. Witten, Nucl. Phys. B 261 (1985) 678.

[12] L. J. Dixon, J. A. Harvey, C. Vafa and E. Witten, Nucl. Phys. B 274 (1986) 285.

[13] A. E. Faraggi, Nucl. Phys. B 387 (1992) 239 arXiv:hep-th/9208024; A. E. Faraggi, Phys. Lett. B 278 (1992) 131; A. E. Faraggi, C. Kounnas, S. E. M. Nooij and J. Rizos, Nucl. Phys. B 695 (2004) 41 arXiv:hep-th/0403058; R. Donagi and A. E. Faraggi, Nucl. Phys. B 694 (2004) 187 |arXiv:hep-th/0403272|; A. E. Faraggi, arXiv:hep-th/0411118. 
[14] A. Font, L. E. Ibáñez and F. Quevedo, Phys. Lett. B 217 (1989) 272.

[15] L. E. Ibáñez, H. P. Nilles and F. Quevedo, Phys. Lett. B 187 (1987) 25.

[16] L. E. Ibáñez, J. Mas, H. P. Nilles and F. Quevedo, Nucl. Phys. B 301 (1988) 157.

[17] H. P. Nilles, arXiv:hep-th/0410160

[18] T. Kobayashi, S. Raby and R. J. Zhang, Phys. Lett. B 593 (2004) 262 arXiv:hep-ph/0403065.

[19] T. Kobayashi, S. Raby and R. J. Zhang, arXiv:hep-ph/0409098. 